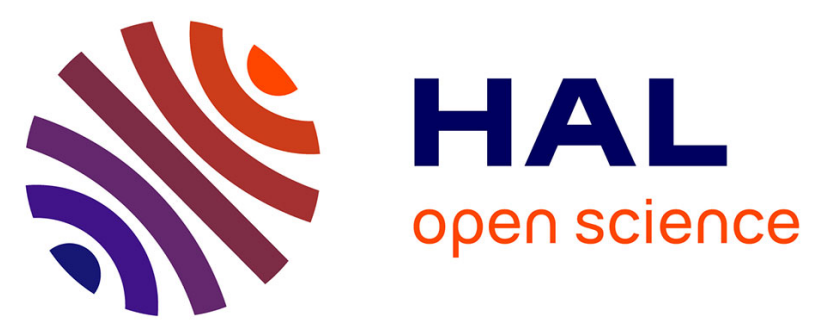

\title{
Exploring the successive waves of cortical folding in the developing brain using MRI and spectral analysis of gyrification
}

Jessica Dubois, David Germanaud, Hugo Angleys, François Leroy, Clara Fischer, Jessica Lebenberg, François Lazeyras, Ghislaine Dehaene-Lambertz, Lucie Hertz-Pannier, Jean-François Mangin, et al.

\section{To cite this version:}

Jessica Dubois, David Germanaud, Hugo Angleys, François Leroy, Clara Fischer, et al.. Exploring the successive waves of cortical folding in the developing brain using MRI and spectral analysis of gyrification. 13th IEEE International Symposium on Biomedical Imaging (ISBI 2016), Apr 2016, Prague, Czech Republic. 10.1109/ISBI.2016.7493259 . hal-01458746

\section{HAL Id: hal-01458746 \\ https://hal-amu.archives-ouvertes.fr/hal-01458746}

Submitted on 6 Feb 2017

HAL is a multi-disciplinary open access archive for the deposit and dissemination of scientific research documents, whether they are published or not. The documents may come from teaching and research institutions in France or abroad, or from public or private research centers.
L'archive ouverte pluridisciplinaire HAL, est destinée au dépôt et à la diffusion de documents scientifiques de niveau recherche, publiés ou non, émanant des établissements d'enseignement et de recherche français ou étrangers, des laboratoires publics ou privés. 


\title{
EXPLORING THE SUCCESSIVE WAVES OF CORTICAL FOLDING IN THE DEVELOPING BRAIN USING MRI AND SPECTRAL ANALYSIS OF GYRIFICATION
}

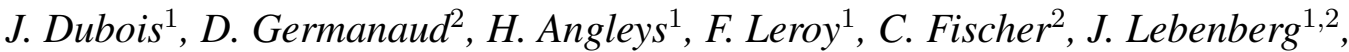 \\ F. Lazeyras ${ }^{3}$, G. Dehaene-Lambertz ${ }^{1}$, L. Hertz-Pannier ${ }^{2}$, J.-F. Mangin ${ }^{2}$, P. Hüppi ${ }^{3}$ and J. Lefèvre ${ }^{4}$ \\ ${ }^{1}$ INSERM-CEA, NeuroSpin Center, Cognitive Neuroimaging Unit U992, Gif-sur-Yvette, France \\ ${ }^{2}$ CEA, NeuroSpin Center, UNIACT (UMR INSERM 1129) and UNATI (CATI), Gif-sur-Yvette, France \\ ${ }^{3}$ Geneva University Hospitals, CIBM and Department of Pediatrics, Switzerland \\ ${ }^{4}$ Aix-Marseille University, CNRS, INT UMR 7289, LSIS, UMR 7296, Marseille, France
}

\begin{abstract}
In the developing human brain, gyrification is a complex process going through the successive appearance of primary folds (from 20 weeks of gestational age GA), secondary folds (from 32 w GA) and tertiary folds (around term age). While this sequence is finely described in fetuses and preterm newborns of different ages using MRI and folding indices, there is still no fully objective assessment of the folding stage at the individual level. We examined the potential of a new method of spectral analysis of gyrification (SPANGY) that was applied to cortical surfaces of 26 preterm newborns, 9 full-term newborns and 17 infants to quantify the spatial-frequency structure of folding. Based on modelling approaches, we unraveled 4 periods along the developmental sequence from 27 to $62 \mathrm{w}$ GA, with relevant timepoints around 31w, 36-38w, and 44-47w GA. These periods showed specific folding features, with spatial patterns of increasing frequencies.
\end{abstract}

Index Terms - Developing brain, gyrification, structural MRI, spectral analysis, primary folds

\section{INTRODUCTION}

In the developing human brain, cortical gyrification is a complex process that mostly takes place during the second half of pregnancy. While the physical or mechanical mechanisms that underlie this process are still debated [1], the folds appearance seems to follow a relatively stable spatio-temporal sequence with three successive waves during development [2] : early primary folds are described from 20 weeks of gestational age (w GA), secondary folds from 32 w GA, and tertiary folds around term age (40w GA) [3]. Since several genetic and environmental factors are likely to influence this process, disturbed gyrification patterns have been observed in many developmental diseases [4]. Understanding their complexity requires to map the folding process in vivo from the early stages, which has become possible in the recent years with non-invasive magnetic resonance imaging (MRI) combined with post-processing tools to extract cortical surfaces [5]. In cross-sectional populations of fetuses and pre-

The research leading to these results has received funding from the Fyssen Fundation, the "Fondation de France", the French National Agency for Research (ANR-12-JS03-001-01, MODEGY), the Geneva CIBM imaging center, the Swiss National Science Foundation (102127; 113632; 33CM30; 135817), the Leenards Foundation, the European consortium NEOBRAIN, and the European Union Seventh Framework Programme (FP7/2007-2013) under grant agreement no. 604102 (Human Brain Project). term newborns, folding features have been measured, showing dramatic increase with age $[6,7]$. Nevertheless there is still no quantitative way to disentangle between primary, secondary and tertiary folds in a single newborn brain. In this perspective, we examined the potential of an original method of spectral analysis of gyrification $(S P A N G Y)$ [8] to describe and quantify the spatial-frequency structure of cortical folding throughout development, from the preterm period to early infancy.

\section{MATERIAL AND METHODS}

\subsection{Methodological background}

SPANGY relies on the spectral decomposition of the mean curvature of the inner cortical surface (i.e. mesh of the grey/white matter interface), based on the Laplace-Beltrami operator eigenfunctions (i.e. a generalization of Fourier analysis to any kind of domain/surface). In this approach, the complexity of the power spectrum is reduced by merging levels of successive orders, assuming a folding model of branching with doublings of spatial frequency [8]. In the adult brain, 7 bands of increasing frequencies (B0-B6) have been shown relevant and sufficient to characterize the curvature patterns in regards to the mesh spatial resolution and the expected size of folding patterns. This approach has further provided an anatomically relevant segmentation of the cortical folds based on the local spectral composition: while the low frequency bands (B0-B3) have been related to the global brain shape, the last 3 bands (B4-B6) have been shown to account for the folds shaping. Furthermore, when the sulci were segmented according to their frequency compound, elements associated with B4, B5 and B6 seemed to match essentially with primary, secondary and tertiary folds respectively. Nevertheless, this anatomical analogy observed in the adult brain remains to be tested throughout the developmental process of folding. We thus applied $S P A N G Y$ over a developmental range crucial for sulci appearance, covering not only the third trimester of pregnancy, but also the first post-natal months.

\subsection{Subjects}

We studied 3 cohorts of babies (all from single pregnancies). First, 26 preterm newborns (11 girls) without neurological complications were imaged within the first two weeks after birth, at GA between $27 \mathrm{w}$ and $36 \mathrm{w}$. Second, 9 full-term healthy newborns (4 girls) were imaged within the first two days after birth, at GA between 
a) Inner cortical surfaces (real proportions across newborns)
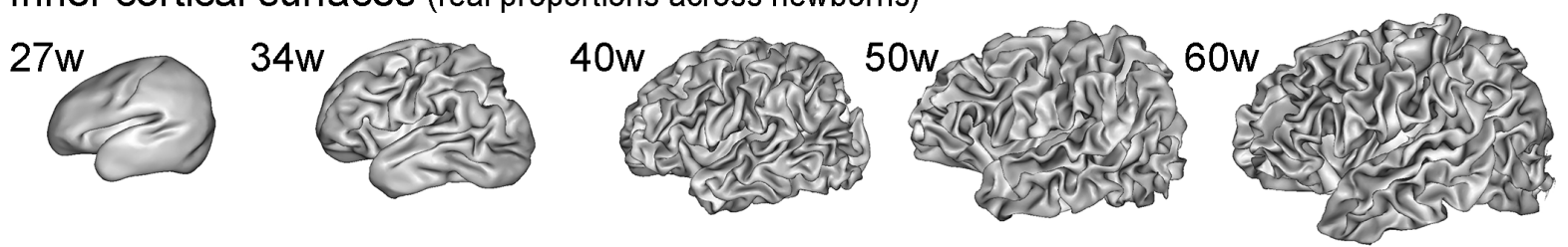

b) SPANGY segmentations projected on smoothed meshes (zoomed proportions)
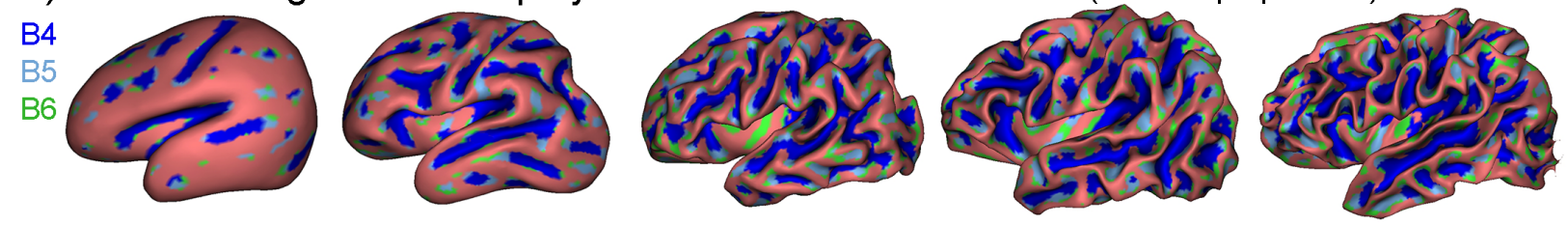

Fig. 1. Cortical surfaces (a) and SPANGY segmentations (b) for 2 preterms, 1 full-term newborn and 2 infants, with regularly sampled ages from $27 \mathrm{w}$ to $60 \mathrm{wGA}$.

38w and 40w. Third, 17 full-term healthy infants (7 girls) were imaged within the first post-natal weeks, at ages (including GA at birth) between $44 \mathrm{w}$ and $62 \mathrm{w}$.

\subsection{MRI acquisition}

High-resolution T2-weighted (T2w) MR images were acquired at $1.5 \mathrm{~T}$ for preterm newborns, and at $3 \mathrm{~T}$ for full-term newborns and infants. Details on preterm and infant data may be found in $[9,10]$ respectively. For full-term newborns, coronal slices were imaged with a spatial resolution of $0.8 \times 0.8 \times 1.2 \mathrm{~mm}(\mathrm{TE} / \mathrm{TR}=150 / 4600 \mathrm{~ms})$.

\subsection{Brain segmentation and morphometric analysis}

The interface between cortex and white matter was segmented using dedicated semi-automatic procedures for preterm newborns [6] and infants [11], with interactive manual corrections performed in the places of low grey/white matter contrast. 3D meshes of the left and right inner cortical surfaces were then provided. Meshes of outer cortical surfaces were also computed from the segmentation of cortex and cerebro-spinal fluid [12]. Among morphological parameters, we measured the area of the inner cortical surface, a sulcation index (defined as the ratio between this area and the surface area after morphological closing), and a proxi of brain size (the hemispheric volume defined as the volume inside the closed outer cortical surface $[8,12])$. Left and right measures were averaged in all analyses.

\subsection{Spectral analysis of cortical curvature}

For each newborn and infant, the mean curvature of the inner cortical surface was computed as implemented in BrainVISA [12]. SPANGY was performed in the same way as in the adult brain, using the previously described Fourier-like analysis [8]. In the spectral domain, total and band B4-B6 spectral powers were computed. In the spatial domain (i.e. on the meshes), we considered a segmentation of cortical sulci (showing negative curvature) according to the locally determinant frequency band [8] : cumulative synthesis was used to label each vertex with the number of the band that determined whether it belonged to the sulcal or the gyral pattern. We then computed the number of parcels of B4 to B6-associated sulci elements.

\subsection{Identification of successive waves of folding}

In addition to age-related changes in whole-brain morphometric parameters, the dependance of cortical surface area on brain size was modelled with a power law. The scaling exponent of the model was compared to the theoretical one under the hypothesis of strictly proportional geometric dependence between surface and volume, to demonstrate non proportional scaling called allometry [7]. Variations in SPANGY parameters with age were further studied in order to identify developmental periods showing specific features of folding or discontinuities. Three complementary approaches were considered.

First, we aimed to evaluate whether spectral bands B4 to B6 captured different temporal mechanisms. For each band, age-related changes in spectral power were modelled with a Gompertz function :

$$
K \exp (-b \exp (-a t))
$$

Gompertz models were preferred to logistic functions because agerelated evolutions were hardly symmetric. Two timepoints of interest were then computed per curve : the inflexion point (corresponding to the vanishing point of the second derivative $: \log (b) / a$ ), and the asymptotic deceleration point (corresponding to the last maximum of the third derivative [13]).

Second, we focused on the proportions between B4-B6 spectral bands through relative spectral powers, defined as the ratios between band powers and total power. For each band, relative power was modelled as the ratio between 2 Gompertz functions for band power and total power. Again, we identified specific timepoints of interest when relevant based on the curves observation.

Third, we aimed to identify distinct developmental periods by integrating the complementary information provided by the three spectral bands. To do so, babies were clustered with a kmeans algorithm applied to B4-B6 relative powers, and we a priori hypothesized 4 groups that should correspond to different ages. Indeed, according to previous observations of 3 successive waves of folding [3], the preterm period should be splitted in 2 sub-periods (before and after 32w GA, when secondary folds start to appear). At term age, when tertiary folds start to appear, another distinct period should be detected. However little is known on the age when folding stops 


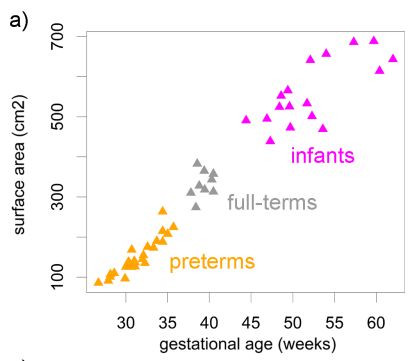

c)

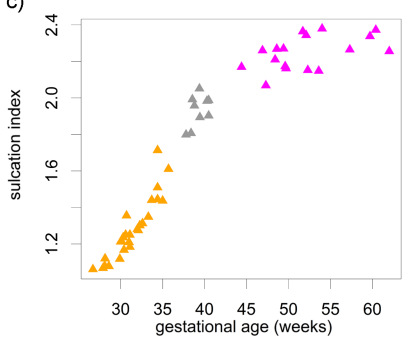

b)

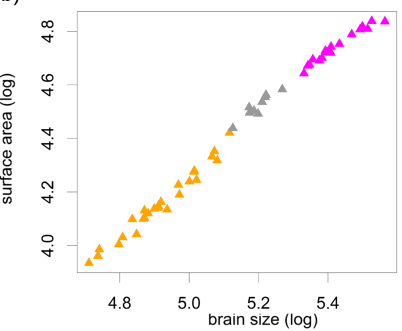

d)

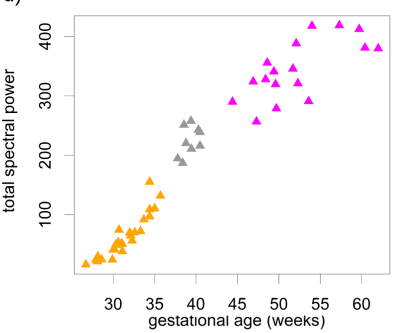

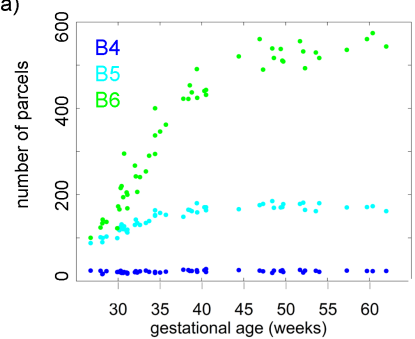

c)

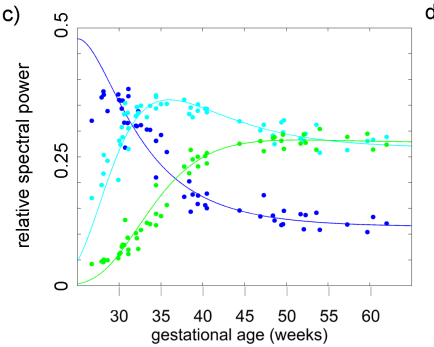

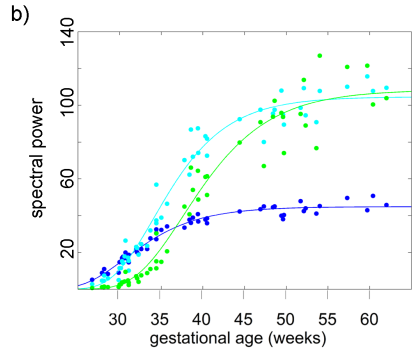

d)

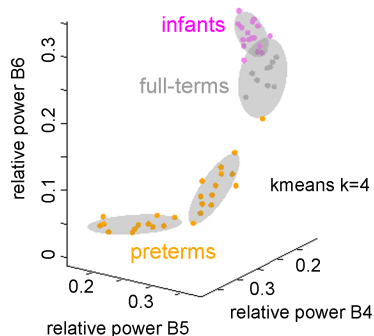

Fig. 2. Variations, across newborns and infants, in whole-brain morphometric parameters : cortical surface area with age (a) or brain size (on a logarithmic scale, b), sulcation index (c) and total spectral power (d) with age.

to proceed, so another developmental timepoint might be relevant during infancy.

\section{RESULTS}

\subsection{Age-related changes in whole-brain parameters}

Inner cortical surfaces were carefully segmented for all preterm, full-term newborns and infants (Figure 1a). Over the developmental period ranging from $27 \mathrm{w}$ to $62 \mathrm{wGA}$, surface area increased significantly with age (Figure 2a), and measures showed high continuity across the 3 groups. As in the adult brain, allometric relationships were observed between surface area and brain size (Figure $\mathbf{2 b}$ ). Nevertheless the observed scaling exponent $\alpha$ was higher than in adults, and seemed to decrease along development (in preterms : $\alpha=1.33$, $\mathrm{R}^{2}=0.9$; in full-term newborns and infants : $\alpha=1.21, \mathrm{R}^{2}=0.93$; vs in adults : $\alpha=0.96$ [8]). Non-linear increases with age were observed for parameters characterizing the folding intensity : sulcation index (Figure 2c) and total spectral power (Figure 2d).

\subsection{SPANGY analyses in the spatial domain : B4- to B6- associated sulci elements}

SPANGY provided a spectral segmentation of cortical sulci which was anatomically coherent over the developmental period Figure 1b). Elements associated with B4 were present in all newborns and infants, overlapping the main earliest folds, whereas elements associated with B5 then B6 increased with age following appearance of branches, dimples and variable folds. These observations could be quantified : whereas the number of B4 parcels was constant over the 27-62w developmental period, the numbers of parcels associated with higher spatial frequencies (B5 and B6 bands) increased dramatically with age (Figure 3a). The number of B5 parcels reached a

Fig. 3. Age-related variations in $S P A N G Y$ B4-B6 characteristics : number of parcels (a), raw (b) and relative (c) spectral powers with Gompertz models. The 3 bands relative powers are also represented in 3D (d), with ellipsoids highlighting the 4 kmeans clusters.

plateau around term age (i.e. 40w GA), earlier than the plateau for B6 parcels (around 45-50w GA).

\subsection{SPANGY analyses in the spectral domain : B4- to B6- spec- tral powers}

Coherently with total spectral power, spectral powers associated with bands B4 to B6 increased significantly over the developmental period (Figure 3b), and all showed a plateau during infancy. However their time courses were different across the bands, as highlighted quantitatively through modelling based on Gompertz functions. Inflexion points were detected at $31 \mathrm{w}, 34 \mathrm{w}$, and $38 \mathrm{w}$ GA for B4, B5 and B6 respectively, suggesting intense folding process during the preterm period. Asymptotic deceleration points were detected at 39w, 43w, and 48w GA for B4, B5 and B6 respectively, highlighting that the folding intensity reached a plateau during early infancy. These results further suggested that the 3 SPANGY bands captured different waves of folding during development.

Second, we observed that the spectral composition changed dramatically but continuously from preterm newborns to infants, as demonstrated by relative spectral powers of bands B4 to B6 (Figure 3c). While preterm newborns showed immature power spectra with high relative power for low spatial frequency (B4 band), full-term newborns and infants already showed adult-like spectral composition with higher relative powers for high spatial frequencies (B5 and mostly B6 bands) than for low frequency (B4 band). From around 30w GA, relative powers continuously decreased for B4 and increased for B6, before reaching plateaus during the late preterm to early term period. Indeed, asymptotic deceleration points were detected at $36 \mathrm{w}$ and $41 \mathrm{w}$ GA for B4 and B6 respectively, according to modelling with ratios of Gompertz functions. Consistently, B5 relative power showed a more complex complementary pattern of changes along development, with a maximum transient plateau at 36w GA. 
To summarize, complex relationships between spectral bands and age were observed, and different timepoints appeared relevant regarding the folding process : around 30w GA (28w-31w GA), around 35w GA (34w-36w GA), around term age (38w-41w GA), and during early infancy (around 48w GA). This is in relative agreement with the previous description of three successive waves of folding along development, with secondary folds appearing from $32 \mathrm{w}$ GA, and tertiary folds around 40w GA [3].

To further define developmental periods with distinct folding features, we finally clustered the newborns and infants in 4 different groups using kmeans algorithm applied to the relative powers of the 3 bands (Figure 3d). This clustering enabled to finely separate the babies in terms of age, with few overlaps across clusters. The first and second clusters gathered almost all preterm newborns over 2 periods : from $27 \mathrm{w}$ to $31 \mathrm{w}$ GA, and from $31 \mathrm{w}$ to $36 \mathrm{w}$ GA respectively. Except one single preterm at $34 \mathrm{w}$ GA with possibly artefactual data, the third and fourth clusters gathered all full-term newborns and infants; the period from $38 \mathrm{w}$ GA was divided in two sub-periods : one below 47w GA, and one above 44w GA (3 overlapping infants). Considering 3 clusters instead of 4 in the kmeans procedure only joined the two latter clusters, with no change in the two former clusters.

\section{DISCUSSION AND CONCLUSION}

In this original study, we analyzed the cortical folding in preterms, full-term newborns and infants with SPANGY, over a developmental period when the progression is intense (from $27 \mathrm{w}$ to $62 \mathrm{w}$ GA). In comparison with previous studies of folding, SPANGY provided a quantitative characterization of the complex spatial patterns of sulci without a priori hypotheses on localization.

Our results supported the relevance of a frequency-based description of the folding sequence along development. It further strengthened the link proposed in the adult brain [8], between the 3 types of spectrally-defined sulci elements provided by SPANGY (associated with bands B4 to B6), and the three types of developmentallydefined folding waves (from primary to tertiary folding), even if this anatomical correspondence cannot be perfect probably due to the process of Fourier-like analysis on complex patterns.

We highlighted that the preterm period is divided in two subperiods, with a relevant timepoint around $31 \mathrm{w}$ GA that corresponds to the appearance of a significant amount of secondary folds. These folds seemed to be finely mapped by the B5 band showing a relative peak around 36w GA. After 38w GA, two additional sub-periods were outlined, that match the appearance of tertiary folds from term age, and the end of the folding process a few weeks after during infancy.

Despite different MRI acquisitions and segmentation postprocessings, high continuity in morphometric and SPANGY parameters was observed across the 3 groups of babies. Notably, the kmeans clustering used to define developmental periods was not driven by the group belonging in a simple way. Nevertheless, some variability was observed across infants, which might rely on inter-individual differences in brain size, for instance in relation to gender.

If these preliminary results were confirmed through longitudinal data of preterms for instance, SPANGY would provide the first quantitative and objective measure of the folding stage at the individual level, in terms of appearance of primary, secondary and tertiary folds.

\section{REFERENCES}

[1] W. Welker, "Why does cerebral cortex fissure and fold? a review of determinants of gyri and sulci," In : Comparative structure and evolution of cerebral cortex. Jones E, Peters A eds. Plenum, New York., vol. Part II, no. vol 8B, pp. 3-136, 1990.

[2] A. Feess-Higgins and J. Laroche Development of the human foetal brain : an anatomical atlas. Inserm-CNRS, Masson, 1987.

[3] J. Chi, E. Dooling, and F. Gilles, "Gyral development of the human brain," Annals of Neurology, vol. 1, no. 1, pp. 86-93, 1977.

[4] J.-F. Mangin, E. Jouvent, and A. Cachia, "In-vivo measurement of cortical morphology : means and meanings," Curr Opin Neurol, vol. 23, no. 4, pp. 359-367, 2010.

[5] J. Dubois and G. Dehaene-Lambertz, "Fetal and postnatal development of the cortex : Mri and genetics.," In : Arthur W. Toga, editor. Brain Mapping : An Encyclopedic Reference. Academic Press : Elsevier, vol. 2, no. 6, pp. 11-19, 2015.

[6] J. Dubois, M. Benders, A. Cachia, F. Lazeyras, R. HaVinh Leuchter, S. Sizonenko, C. Borradori-Tolsa, J.-F. Mangin, and P. S. Hüppi, "Mapping the early cortical folding process in the preterm newborn brain," Cerebral Cortex, vol. 18, no. 6, pp. 1444-1454, 2008.

[7] J. Lefèvre, D. Germanaud, J. Dubois, F. Rousseau, I. de Macedo Santos, H. Angleys, J.-F. Mangin, P. S. Hüppi, N. Girard, and F. De Guio, "Are developmental trajectories of cortical folding comparable between cross-sectional datasets of fetuses and preterm newborns ?," Cerebral Cortex, vol. in press, p. pii : bhv123, 2015.

[8] D. Germanaud, J. Lefèvre, R. Toro, C. Fischer, J. Dubois, L. Hertz-Pannier, and M. Jean-François, "Larger is twistier : spectral analysis of gyrification (spangy) applied to adult brain size polymorphism," Neuroimage, vol. 63, no. 3, pp. 12571272, 2012.

[9] J. Dubois, M. Benders, F. Lazeyras, C. Borradori-Tolsa, R. Leuchter, J.-F. Mangin, and P. S. Hüppi, "Structural asymmetries of perisylvian regions in the preterm newborn," $\mathrm{Neu}$ rolmage, vol. 52, no. 1, pp. 32-42, 2010.

[10] C. Kabdebon, F. Leroy, H. Simmonet, M. Perrot, J. Dubois, and G. Dehaene-Lambertz, "Anatomical correlations of the international 10-20 sensor placement system in infants," Neuroimage, vol. 99, pp. 342-356, 2014.

[11] F. Leroy, J.-F. Mangin, F. Rousseau, H. Glasel, L. HertzPannier, J. Dubois, and G. Dehaene-Lambertz, "Atlas-free surface reconstruction of the cortical grey-white interface in infants," PLoS ONE, vol. 6, no. 11, p. e27128, 2011.

[12] C. Fischer, G. Operto, S. Laguitton, M. Perrot, I. Denghien, D. Rivière, and J.-F. Mangin, "Morphologist 2012 : the new morphological pipeline of brainvisa," NeuroImage, vol. Proceedings of HBM Scientific Meeting, no. Beijing, China, p. 670, 2012.

[13] M. Mischan, S. Zambello de Pinho, and L. de Carvalho, "Determination of a point sufficiently close to the asymptote in nonlinear growth functions," Scientia Agricola, vol. 68, no. 1, pp. 109-114, 2011. 